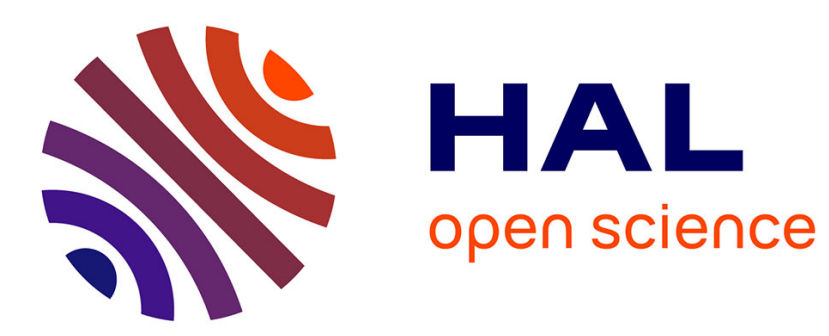

\title{
Telerobotic Surgery: Fuzzy Path Planning Control for a Telerobotic Assistant Surgery
}

Rahma Boucetta

\section{To cite this version:}

Rahma Boucetta. Telerobotic Surgery: Fuzzy Path Planning Control for a Telerobotic Assistant Surgery. 14th Computer Information Systems and Industrial Management (CISIM), Sep 2015, Warsaw, Poland. pp.297-304, 10.1007/978-3-319-24369-6_24 . hal-01444473

HAL Id: hal-01444473

https://hal.inria.fr/hal-01444473

Submitted on 24 Jan 2017

HAL is a multi-disciplinary open access archive for the deposit and dissemination of scientific research documents, whether they are published or not. The documents may come from teaching and research institutions in France or abroad, or from public or private research centers.
L'archive ouverte pluridisciplinaire HAL, est destinée au dépôt et à la diffusion de documents scientifiques de niveau recherche, publiés ou non, émanant des établissements d'enseignement et de recherche français ou étrangers, des laboratoires publics ou privés. 


\title{
Telerobotic Surgery: Fuzzy Path Planning Control for a Telerobotic Assistant Surgery
}

\author{
Rahma Boucetta \\ Laboratory on Control and Energy \\ Management (CEM Lab) \\ National Engineering School of Sfax \\ University of Sfax \\ BP W, 3038 Sfax, Tunisia \\ Email: boucetta.rahma@gmail.com
}

\begin{abstract}
A strategy of path planning with fuzzy logic for a telerobotic assistant surgery is presented in this paper. Telerobotic surgery occurred a long track in its short history. While teleconsultation proceeds to be used today, the advent of high speed communications and increased computational competence is making long distance remote control of operating instruments, called telepresence surgery, a reality. Based on laparoscopic technology, telerobotic surgery was tested first on animals and, more recently, on humans with success. The technology offers several advantages, including improved accuracy and the capability to bring difficult procedures to rural and remote locations where trained surgeons are not available. A dynamic model is computed first for the telerobots using Lagrange formulation. A fuzzy control strategy is used in order to validate the path planning method and the theoretical developments in motion constraints analysis. The paper is ended with a conclusion.
\end{abstract}

Keywords: Telerobotic Surgery, Dynamic Model, Path Planning, Fuzzy Logic, Laparoscopic Surgery.

\section{Introduction}

Surgery has evolved from the early rough days of trepanation and battlefield amputations to modern procedures such as complex neurosurgeries and minimally invasive laparoscopic interventions. However, the surgeon is virtually useless without his tools, and it is perhaps the development of these tools, which has directly lead the evolution of surgery. While several tools such as scalpels, stitches, loops, anaesthesia and antiseptics have each amplified the set of possibilities for procedures, some of the newest tools to enter the surgical instrumentation are robots and computers.

While the advantages of computer and robotic assistance in surgery, in terms of improved accuracy and control may seem evident, one of the most interesting and useful applications of this technology is to perform surgeries remotely. Patients in rural and remote locations often do not have access to advanced 
surgical care due to an absence of qualified personnel [1]. This is the case in both wartime battlefields and third world countries, and many remote areas as Canada country. Surgical care in these emplacements is either impossible or requires transportation to an urban center upon long distances.

The prime essays at remote care were really what would be appealed teleconsultation. The 1960s saw the beginning of electronic transmission of radiological films, while the 1970s brought the ability for medicaster to consult with experts remotely over video-conference systems [3]. In a surgical implementation of teleconsultation, a remote video-conferencing system was set up in the operating room and was linked to an expert physician at an urban center [2]. This apart surgeon did not actively participate in the process, but instead suggested advice or guidance to the attending surgeon at critical points. At best, electronic remote control of the video camera was available, but little else. While accurate benefits in terms of transmitting expertise and training inexperienced surgeons could be realized with this setup, true remote surgical control was impossible. This changed with the advent of a robotic system tested at assisting in laparoscopic procedures. Laparoscopic surgery uses a miniature camera (i.e., laparoscope) and small surgical tools which are introduced into the body via tiny incisions and controlled through external manipulators. Minimally invasive surgery performed using laparoscopy provided various advantages to the patient : less pain, a shorter hospital stay, better cosmetic outcome and faster recovery [4]. Unfortunately, this surgical technique, in its original conception, had several shortcomings. The laparoscope produced only a 2 -dimensional view of the surgical area, and hand-eye coordination was difficult due to the need to look at a monitor instead of ones hands. Even, the laparoscope was held by an assistant, and therefore direct was taken out of the hands of the surgeon. Perhaps most importantly yet, laparoscopy, by its very nature, introduced amplification of vibration, loss of degrees of freedom (dof) in manipulation, and the brought the requirement for making non-intuitive motions when performing a process [4]. To cross the inherent limitations of laparoscopic surgery, research supported by the US Defense Departments Star Wars program was undertaken in the early 1990's to develop a master-slave tele-manipulator, consisted on a computer and robotic instrumentation intervened between the surgeon and the patient [4]. The original goal of this technology was to allow actual manipulation of surgical instruments by telepresence surgery [5]. This technology is hoped to be useful in performing remote trauma surgeries on the battlefield or outer space, where surgeons could not venture [5]. Unfortunately, while a system was developed, it missed the required dof necessary for efficient surgery, and its large size stopped expanded use.

In 1994, a private company developed the da Vinci robot system. This robot builds on traditional laparoscopic technology, rectifying some of its faults while introducing the capacity for remote manipulation. The first refinement is that the camera platform is stable, and can be controlled by the surgeons feet or voice commands, eliminating the need for an assistant. Second, visualization is greatly enhanced with a 3-dimensional magnified system to simulate natural vision, or 
alternately 2-dimensional displays positioned near the hand controls [6]. Moreover, since physical manipulation of the controls is processed by a computer, shaking can be digitally filtered out preventing excessive error. Finally, the use of motion scaling, which reduces large movements to fine ones, allows surgeons to perform actions which were previously impossible due to their delicacy [6]. Early telepresence surgery research was extremely limited, and being hampered by technical limitations, was carried out only on animal models. Advanced manipulation techniques were not possible due to lack of adequate computational power and communication bandwidth. An early procedure was performed in 1993 by issuing keyboard and mouse commands to manipulate an echographic sonde, biopsy needle and scalpel over a transatlantic fiber optic telephone link to remove a cyst from a pigs liver. Unfortunately, transfer of real-time video over the wired network was technologically impossible at the time due to bandwidth constraints; consequently, relatively expensive satellite links were required [2]. One of the leading difficulties in developing clinically viable telerobotic surgery has been the requirement of minimal time lag between the issuing of commands, actual surgical action, and reception of visual confirmation on the screen. This lag is influenced by multiple factors including time required for converting video and movements into the appropriate signals and the inherent delay in the communication network itself. Experiments have determined that the acceptable limit for safe surgery is $330 \mathrm{~ms}$ [7]. Even with the satellite video link in early experiments, overall delays of approximately $2 s[2]$ were inherent in the technology, obviously far from acceptable for a real-time surgical procedure. Accordingly, it was estimated that feasible distances for remote surgery could not exceed several hundred kilometers [8]. This was, however, disproved in subsequent years.

This paper is composed of the following sections : after an introduction, the dynamic model is computed in the second paragraph using the Lagrange equations. The development of a fuzzy path planning control is given by the next section to analyse the telerobotic surgery behavior. the paper is ended with a conclusion.

\section{Dynamic Modelling of the Surgical Robot}

\subsection{Concepts}

Safety, control convenience, and flexibility for use in an ample variety of surgical applications were important factors in determining the manipulator design. In laparoscopic applications, rigid instruments are inserted into the patient's body through small cannulas slotted into the abdominal wall. This settlement creates a swivel effect, so that the instrument has only four significant motion dof (three rotations and depth of penetration) centered at the gate. Only very constrained lateral motions are acceptable.

If a robot is keeping an instrument, its motions obey these constraints, necessary. A conventional industrial robot can, of course, be programmed move an instrument about such a fulcrum. Unfortunately, such motions usually require a lot of manipulator joints to make large, tightly coordinated excursions. Thus, 
even relatively slow end-effector motions can require rapid joint motions. Any control or coordination failure can thereby represent a potential safety hazard both for the patient and for the surgeon. Simply slowing down the actuators can cause the entire functioning of the robot to be painfully boring. Consequently, manipulator designs that require only low velocity actuation are preferred, they do not have motion singularities in the normal working volume, and permit simple stable controls. Similarly, the motions required to perform a task should be reasonably intuitive for the surgeon. Even if the control computer is handling all the details, it is desirable not to surprise the surgeon with unanticipated complex motions. Finally, a great agreement of modularity allow us to reconfigure the system for different procedures.

The 4 dof Robotic Arm chosen in our study delivers fast, accurate, and repeatable movement. The robot features are the Z-base rotation, the single X-plane shoulder, the $\mathrm{X}$-elbow, the $\mathrm{X}$-wrist motion, and a functional gripper.

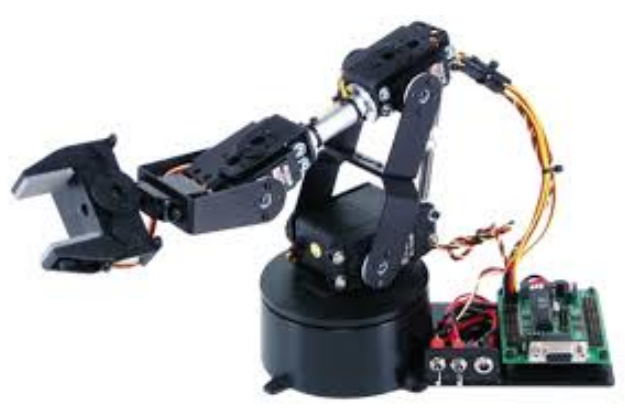

Fig. 1. 4-dof Surgical Robot architecture.

The dynamic model of the surgical robot was developed using the Lagrange formulation. The robot is described by Figure 1, using a right-handed inertial frame $\left(B_{0}-\right.$ frame $)$ represented by $\left(X_{0}, Y_{0}, Z_{0}\right)$, and three right-handed body frames $\left(B_{i}-\right.$ frame $)$ represented by $\left(X_{i}, Y_{i}, Z_{i}\right)$, where $i$ is the number of dof $i=1 . .4$. The positive direction of the first angle $\theta_{1}$ is decided by a right handed rotation about positive $Z$ axis, the others angles $\theta_{2}, \theta_{3}$ and $\theta_{4}$ are about $X$ axis respectively.

Each vector $V_{0}$ in the $B_{0}-$ frame can be transformed into a vector $V_{i}$ in the $B_{i}-$ frame by the following relation

$$
q_{0}=R_{0, i} \cdot q_{i}
$$




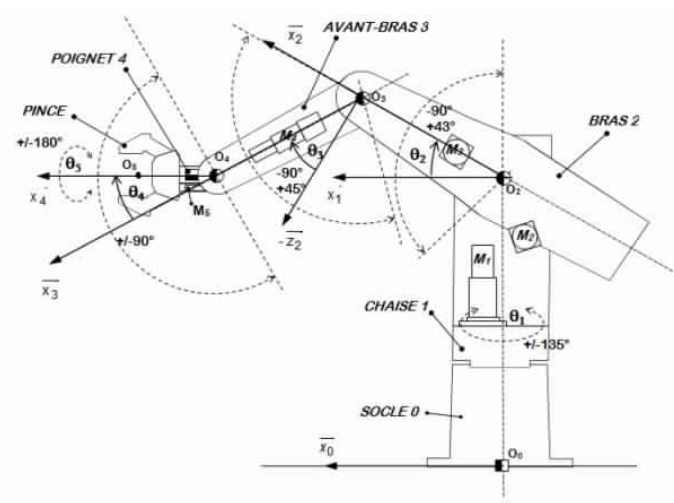

Fig. 2. Cinematics of the Surgical Robot.

where $R_{0, i}$ is the rotation matrix of the $B_{i}-$ frame relative to the $B_{0}-$ frame determined as

$$
R_{0,1}=\left(\begin{array}{ccc}
C_{1} & -S_{1} & 0 \\
S_{1} & C_{1} & 0 \\
0 & 0 & 1
\end{array}\right), R_{i, i+1}=\left(\begin{array}{ccc}
1 & 0 & 0 \\
0 & C_{i+1} & -S_{i+1} \\
0 & S_{i+1} & C_{i+1}
\end{array}\right)
$$

\subsection{Dynamics}

The dynamic behavior can be generated using the Lagrange formulation given by the expression as

$$
\frac{d}{d t}\left(\frac{\partial L}{\partial \dot{q}_{i}}\right)-\frac{\partial L}{\partial q_{i}}=\Gamma
$$

where $L$, the lagrangian, that is the difference between kinetic and potential energies given by $L=T-V . T$ is the kinetic energy of the overall system and $V$ is the potential energy due to the internal deformation of the robot that is equal to zero. So, the Lagrangian becomes equal to the sum of the kinetic energies of the moving bodies. The kinetic energy of each body with mass $M_{i}$ has the general expression as follows

$$
T_{i}=\frac{1}{2}\left(M_{i} V_{G_{i}}^{2}+\Omega_{i}^{T} I^{(i)} \Omega_{i}\right)
$$

where $V_{G_{i}}$ is the translation velocity vector of the center of inertia of the moving link $i$ and has the following form

$$
V_{O_{i+1}}^{(0)}=V_{O_{i}}^{(0)}\left(R_{i}\right)+\Omega_{i}^{(0)}\left(R_{i}\right) \wedge \overrightarrow{O_{i} O_{i+1}}\left(R_{i}\right)
$$


and $\Omega_{i}^{(0)}\left(R_{i}\right)$ is the rotation velocity vector given by

$$
\Omega_{i}^{(0)}\left(R_{i}\right)=\breve{\Omega}_{i}^{(i-1)}\left(R_{i-1}\right)+\left[R_{i-1, i}\right]^{T} \Omega_{i-1}^{(0)}\left(R_{i-1}\right)
$$

The dynamic model can be deduced from the computation of the kinetic energies and the derivation of Lagrange equations. It has the following expression

$$
M \ddot{\theta}+K \dot{\theta}^{2}+C \dot{\theta} \dot{\theta}=\Gamma
$$

where $M, K$ and $C$ are the inertial, centrifugal and coupling forces matrices, respectively. To simulate the surgical robot model, the real dimensions values are given as $L_{2}=0.175 \mathrm{~m}, L_{3}=0.28 \mathrm{~m}, M_{2}=0.64 \mathrm{~kg}$, and $M_{3}=0.6 \mathrm{~kg}$.

\section{Fuzzy Path Planning Control}

A fuzzy controller (FC) consists of a set of linguistic conditional statements that are derived from human operators, and which represent knowledge about the system being controlled. These statements define a set of control actions using if-then rules. The FC can be considered as a fuzzy reasoning process to manifest itself in the form of qualitative information about the process, and this information is articulated in the if-then rules, [9].

The first step to be performed by the FC is to fuzzify each input, which is done by associating each input with a set of fuzzy variables. In order to give semantics of a fuzzy variable a numerical sense, a membership function is assigned to each variable. The form of a membership function can be either discrete or continuous, and its range is varied from zero to one. Two types of continuous membership functions commonly adopted in fuzzy logic control are triangle and exponential forms. Fuzzified inputs are then associated with knowledge base of the fuzzy controller, containing a set of control rules. By matching the fuzzified inputs with each control rule activates a set of control actions. Since the control actions are in the fuzzy sense, defuzzification is required to transform fuzzy control actions into an exact output value of the controller, [9].

3 illustrates the block diagram of a direct fuzzy controller applied to the flexible manipulator. Control rules process the error between hub angle and its desired value (e) and the change in error (ce) to synthesize a change in control for improving system performance and vibration suppression. Membership functions are chosen triangular and symmetric. The universes of discourse are divided to 7 fuzzy sets for the inputs and 7 fuzzy sets for the output and chosen between $[-1,+1]$. So, scaling gains are introduced to normalize the control system. Knowledge rule-base is composed of $7 \times 7=49$ rules. 


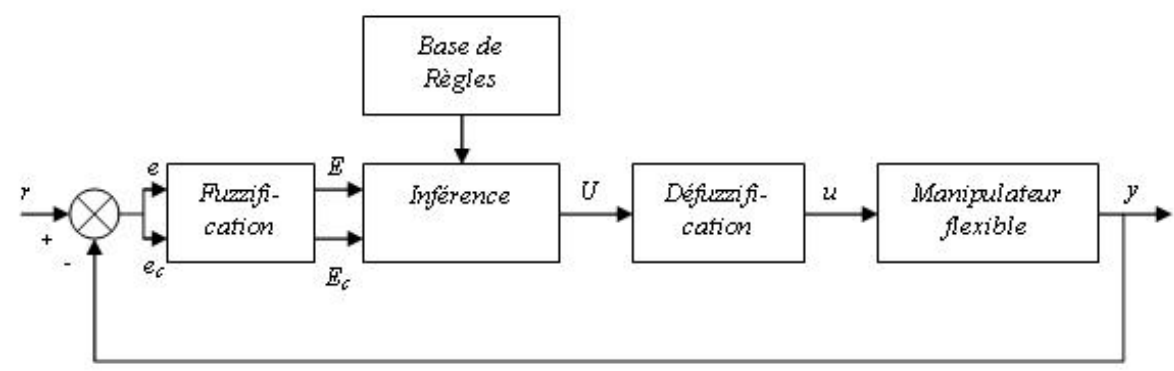

Fig. 3. Diagram of Fuzzy Controller.

\section{Simulation Results}

The models of master-slave surgical robots are inserted in a simulink file. The fuzzy controllers are then established and placed in the closed loop of the robots. The consign signals are chosen to put the end-point in a desired position. The slave receive the same information after a second. Simulation results are given by the next figures 4,5 and 6 .

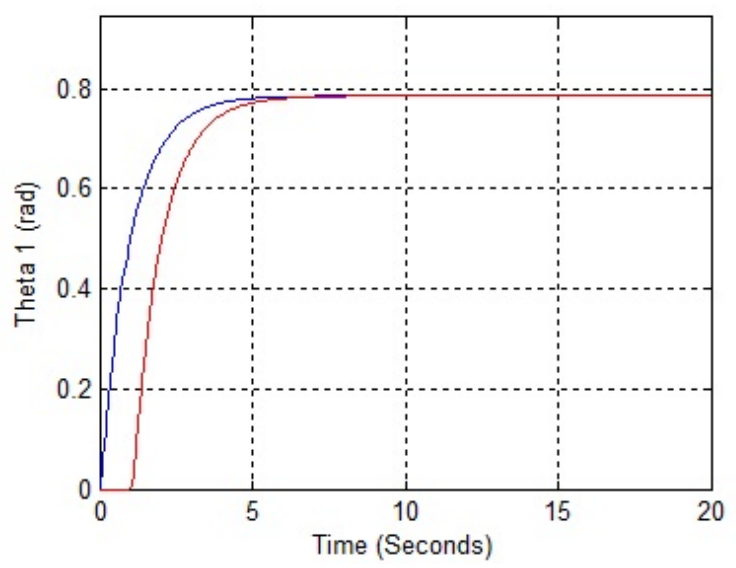

Fig. 4. Responses and first angle $\theta_{1}$.

The first Z-angle $\theta_{1}$ for the master and the slave robots are stables and reach consign slowly without overshoots. The second X-angle $\theta_{2}$ is more fast for the master robot and slow with some fluctuations for the slave robot. The third $\mathrm{X}$-angle $\theta_{3}$ is very fast with some overshoots for the master robot, and slow and stable for the slave robot. The slave robot can easily pursuit the master robot 


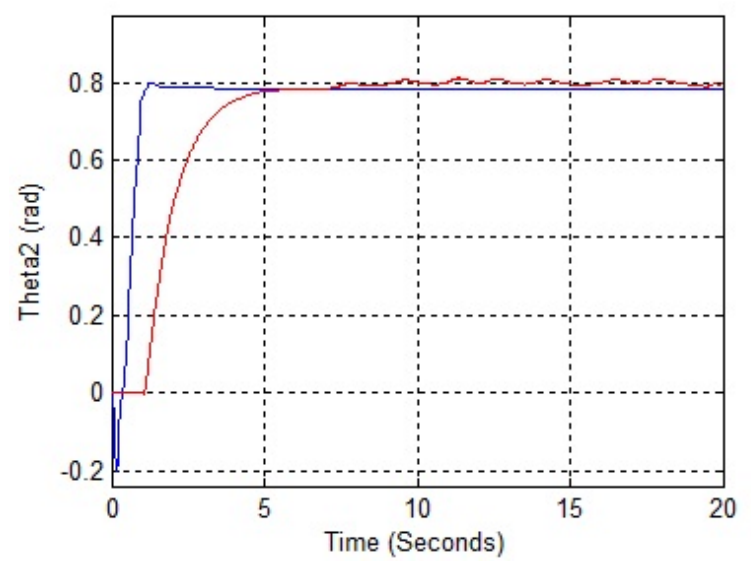

Fig. 5. Responses and second angle $\theta_{2}$.

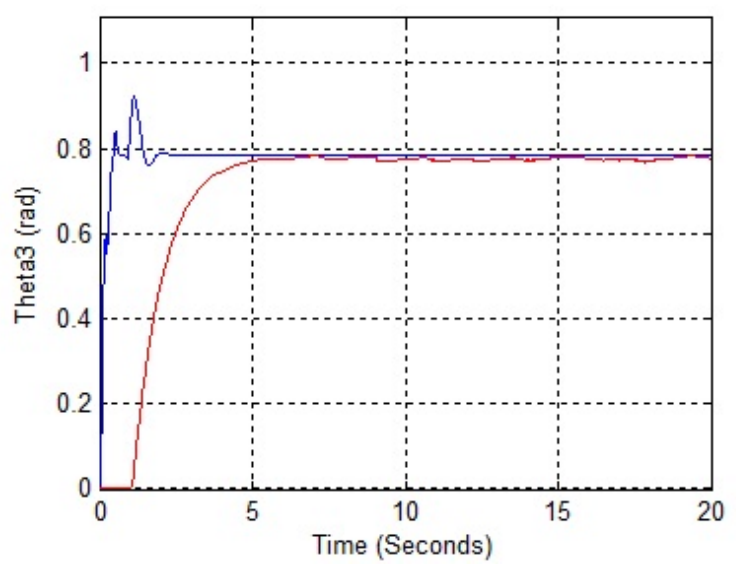

Fig. 6. Responses and third angle $\theta_{3}$. 
in its path planning in spite of the delay between the robots operations.

\section{Conclusion}

This paper deals with telerobotic surgery. The main task is to determine a dynamic model of a serial surgical robot using the Lagrange formulation. A fuzzy path planning control is then developed to command a master-slave robot for a surgery application. Simulations results are given to compare different outputs variations. This work can be improved with other types of controllers and real environment in the near future.

\section{References}

1. Anvari, M.: Reaching the rural world through robotic surgical programs. Eur. Surg. 37(5), 284-292 (2005)

2. Rovetta, A.: Remote Control in Telerobotic Surgery. IEEE Transactions on Systems, Man and Cybernetics. 26(4), 438-444 (1996)

3. Benger, J.: A review of telemedicine in accident and emergency: The story so far. Emerg. Med. J. 17, 157-164 (2000)

4. Broeders, I., Ruurda, J.: Robotics revolutionizing surgery: the Intuitive Surgical "Da Vinci" system. Industrial Robot. 28(5), 387-391 (2001)

5. Bowersox, J., Cordts, P., LaPorta, J.: Use of an intuitive telemanipulator system for remote trauma surgery: An experimental study. J Am Coll Surg. 186(6), 615-621 (1998)

6. Costello, A. Haxhimolla, H., Crowe, H. Peters, J.: Installation of telerobotic surgery and initial experience with telerobotic radical prostatectomy. BJU Int. 96, 34-38 (2005)

7. Marescaux, J., Leroy, J., Gagner, M., Rubino, F., Mutter, D., Vix, M. Butner, S., Smith, M.: Transatlantic robot-assisted telesurgery. Nature. 413, 279-380 (2001)

8. Marescaux, J., Rubino, F.: Robotic surgery: potentials, barriers and limitations. Eur. Surg. 37(5), 279-283 (2005)

9. Passino, K.M., Yurkovich, S.: Fuzzy Control. Addison-Wesley, Longman (1998) 